\title{
sciendo
}

\section{Inter-Session Reliability of the Tennis Serve and Influence of the Laboratory Context}

\author{
by \\ François Tubez ${ }^{1,2,3}$, Bénédicte Forthomme ${ }^{1,2}$, Jean-Louis Croisier ${ }^{1,2}$, \\ Olivier Brüls ${ }^{1,4}$, Vincent Denoël1,5, Julien Paulus ${ }^{1}$, Cédric Schwartz ${ }^{1}$
}

To meet the demand of a player's entourage (e.g., coaches and medical staff), it is important for the biomechanics specialist to perform repeatable measures. To the best of our knowledge, to date, it has not been demonstrated whether similar results are obtained between two sessions of testing or between laboratory and field sport kinematic protocols with regard to the tennis serve. This study had two primary aims. First, the inter-session repeatability of biomechanical variables of a tennis serve was evaluated. Second, the differences between laboratory and field evaluations were studied. Thirteen national tennis players (ITN 3) performed the same 28 markers' set laboratory test twice two weeks apart, and other thirteen national players (ITN 3) performed two 4 markers' set tests both in the laboratory and on an official tennis court one week apart. A 3D motion system was used to measure lower-limb, pelvis, trunk, dominant arm and racket kinematics. A force plate was used to evaluate kinetics of legs' drive in the laboratory. A personal method based on a point scoring system was developed to evaluate the ball landing location accuracy. We observed that the majority of the studied variables were acceptable for excellent relative reliability for the inter-session analysis. We also showed that the impact of the laboratory versus field context on the player's serve was limited.

Key words: repeatability, sport, protocol, test-retest, biomechanics.

\section{Introduction}

Current technologies influence sport performance by providing specific and accurate physical and physiological information. In the tennis biomechanics area, kinetic and kinematic variables are regularly considered for performance enhancement as well as injury prevention (Kovacs and Ellenbecker, 2011). For example, legs drive (Girard et al., 2007), trunk rotations (Bahamonde, 2000), ball accuracy (Göktepe et al., 2009), racket velocity and height at impact (Martin et al., 2014; Whiteside and Reid, 2016) are described in the literature as performance variables, while dominant shoulder internal and external rotations (Elliott et al., 2003)

or trunk rotations (Martin et al., 2014) could intervene in injury mechanisms.

To meet the demands of a player's entourage (e.g., coaches and medical staff), it is important for the biomechanics specialist to perform repeatable measures and subsequently provide efficient longitudinal follow-up evaluations to the player. Thus, effect of training or rehabilitation sessions can be observed through repeated evaluations. The reliability of kinetic and kinematic measurements has already been determined in the literature for the gait (Manca et al., 2010), but to the best of our knowledge, there has been no study conducted on the tennis serve

\footnotetext{
1 - Laboratory of Human Motion Analysis, University of Liège, Liège, Belgium.

2 - Department of Sciences and Motor Skills, University of Liège, Liège, Belgium.

3 - Physiotherapy Department, Haute École Robert Schuman of Libramont, Libramont, Belgium.

4 - Department of Applied Sciences, University of Liège, Liège, Belgium.

5 - Department of Aerospace and Mechanical Engineering, University of Liège, Liège, Belgium.
} 
in terms of biomechanical evaluation. The main literature on test-retest situations in tennis is described in terms of groundstroke evaluations and not of a biomechanical evaluation (Ferrauti et al., 2011).

The laboratory context allows performance of biomechanical evaluations with maximally controlled conditions, while numerous uncontrollable external variables influence field situations. However, what is observed in the laboratory might not be transposable to the real tennis court context because the physical environment and psycho-emotional feelings differ markedly between these two contexts of testing. It is important to consider this question as many publications in the field of sports are based on a laboratory context and not the actual sports field (Tubez et al., 2015, 2017). The agreement between the laboratory and the sports field has already been studied and reported in the literature for cycling physiology (Karsten et al., 2014) and softball performance (Smith and Kensrud, 2014). On the contrary, in soft-tennis, the kinematic and kinetic characteristics of the smash under match conditions differ from those observed under laboratory-simulated conditions (Ida et al., 2005). To the best of our knowledge, it has not been demonstrated to date whether similar results are obtained for laboratory and field tennis serve kinematic protocols comparisons, although we could expect repeatable measurements because the tennis serve is a closed skill.

This study had two main aims. First, the inter-session repeatability of biomechanical variables of a tennis serve was evaluated. Second, the differences between laboratory and field evaluations were studied. We focused on variables regularly used in the literature and dedicated to tennis serve evaluation. Variables determined to be reliable between two sessions and between the two contexts (laboratory versus tennis court) could be used in the future by biomechanics specialists.

\section{Methods}

\section{Participants}

Our study was divided into two parts. The first part focused on the reliability of measures under laboratory conditions. Thirteen players $(22 \pm 3$ years, $180 \pm 5 \mathrm{~cm}, 71 \pm 7 \mathrm{~kg}$ ) performed the same 28 markers' set laboratory test twice two weeks apart. The second part focused on the influence of the laboratory versus the tennis court context on the characteristics of the serve. Thirteen players distinct from the first part of the study ( $23 \pm 3$ years, $180 \pm 4 \mathrm{~cm}, 75 \pm 7$ $\mathrm{kg}$ ) performed two 4 markers' set tests both in the laboratory and on an indoor official tennis court one week apart. For both parts of the experiment, the players were men without injuries who were right-handed and advanced tennis players ranked International Tennis Number 3.

The reported protocol was approved by the Medical Ethics Committee of the University of Liège (Belgium). All players provided written informed consent in accordance with the Declaration of Helsinki.

\section{Instrumentation}

An optoelectronic system based on four Codamotion CX1 units (Codamotion ${ }^{\mathrm{TM}}$, Charnwood Dynamics, Rothley, UK) was used in the laboratory and on the court to measure 3dimentional kinematic variables of the lower limbs, trunk, dominant arm and racket. The acquisition rate was equal to $200 \mathrm{~Hz}$.

Depending on the test, twenty-eight or four active markers were used. For the 28 markers' set (Figure 1), four markers were placed on the dominant arm, four on the dominant forearm and two on the dominant hand (Schwartz et al., 2016). Two markers were placed on both sides of the racket, and one was placed on the top (Whiteside et al., 2013b). The barycentre of the racket was considered to be at the center of these markers. Four markers were placed on both legs, four on the pelvis and four on the trunk. The trunk markers were placed in accordance with the International Society of Biomechanics (Wu et al., 2005). A pointer was employed to digitalize the following additional anatomical landmarks: D8, left and right posterior-superior iliac spine, right lateral epicondyle, right medial epicondyle joints. The center of the right glenohumeral joint was calculated using the method proposed by Gamage and Lasenby (2002). For the tests, all of the players used their own racket. With regard to the 4 markers' set protocol, only the three racket markers and the wrist marker were used. Only 4 markers were used for the field test to have a comparison closer to the real game (less markers on the body) and limit the possible influence of the extended marker set test. 
Part of a tennis court (Figure 2) was reproduced in the laboratory (Tubez et al., 2015). The width of the court was smaller $(5.8 \mathrm{~m})$ than the normal size $(8.23 \mathrm{~m})$ in order to accommodate the laboratory conditions, yet the distance to the square (length) was the same as in an official tennis court. The net was placed at the distance and height specified under tennis regulations (International Tennis Federation, Roehampton, England).

A one-square-meter target was positioned at the " $\mathrm{T}$ " zone of the deuce square (Martin et al., 2014). This procedure is in accordance with the characteristics described by Whiteside and Reid (2016) who proposed to serve in this area to perform an ace.

A personal method based on a point scoring system, as encountered in the literature, was developed to evaluate the ball landing location accuracy (Figure 3) (FernandezFernandez et al., 2014). The closer the ball was to the "T zone", the more points were given.

\section{Design and procedures}

Each player performed a personal ten minute general warm-up using elastic bands and a jump rope. A five-minute specific warm-up composed of tennis serve trials was also realized by each player. This warm-up was conducted as during tennis training or a match (Reid et al., 2010; Whiteside et al., 2013a). This period also allowed the players to familiarize themselves with the laboratory and the target area. Thereafter, the markers were placed on the skin by an experiment operator. The players were later allowed to conduct an additional five minute serve practice. The duration of the warm-up was set in order to avoid fatigue. The serve evaluation started after the familiarization. The evaluation consisted of 15 trials of a tennis serve in the direction of the " $\mathrm{T}$ " area with $30 \mathrm{~s}$ rest intervals between each trial. Instructions were "to serve in the one square meter zone with the highest racket speed and minimal ball rotation, as in a flat serve" (Bahamonde, 2000).

\section{Data reduction and analysis}

The three best serves of each test were selected (Mullineaux et al., 2001). Selections were first based on the ball landing location (in the onesquare-meter zone) and second on the racket speed at impact. If the number of serves selected based on these criteria were not sufficient, serves close to the $1-\mathrm{m}^{2}$ zone with a high racket speed were taken into account. For the accuracy variable only, we analyzed all 15 serves. We set the $\mathrm{x}$-axis as antero-posterior in the direction of the net, $y$ axis as parallel to the baseline in the direction of the right and the z-axis as vertical in the direction of the top.

With $X$ being the antero-posterior direction, $\mathrm{Y}$ being the lateral direction and $\mathrm{Z}$ the infero-superior direction, orientations of the pelvis relative to the laboratory, of the thorax relative to the pelvis, of the arm relative to the thorax, of the forearm relative to the arm, of the wrist relative to the forearm and of the racket relative to the laboratory were evaluated using ZYX, YXZ, XZY (Bonnefoy et al., 2010), ZXY (Wu et al., 2002), XYZ (Wu et al., 2002), ZXY Cardan decomposition, respectively. To compare the bone rotations of different subjects, a unique humeral reference position was defined: all humeral rotations were set to $0^{\circ}$ for the initial posture, with the arms along the body and the elbow being flexed at $90^{\circ}$.

\section{Variables and time points of interest}

The serve action is a succession of key events and phases (Giblin et al., 2017; Whiteside et al., 2013b). Propulsion is the phase delimited with the high racket point (HRP or trophy position) and the low racket point (LRP). Forward swing is the phase between the LRP and impact. To compare the serves, impact was chosen as the reference instant $($ time $=0.0 \mathrm{~s})$.

The variables of interest were those considered important in the literature to generate racket velocity and are described hereafter (Whiteside et al., 2013b). The serve starts with the player's leg drive during the propulsion phase. The energy from the legs is then transmitted through the body up to the racket to finally reach the ball at impact. The distance between the feet (foot technique) is calculated between both hallux markers at the leg drive instant. Peak knee flexion indicates the magnitude of preparation before the leg drive. Lower limb triple extension (combination of ankle, knee and hip extension velocities) (Whiteside et al., 2013b) is the kinematic representation of the leg drive propulsion. The action of the leg drive can also be evaluated through the ground reaction forces. Energy is transmitted from the legs to the trunk through the pelvis. The consequence of this 
transmission is partly represented with vertical back hip velocity (Tubez et al., 2015). The peak separation angle and peak trunk-pelvis angle represent the angles between the shoulder and the pelvic girdles, respectively, in the frontal and transversal planes. These findings indicate the magnitude of preparation of the trunk to transmit the energy flow to the dominant arm. Trunk twist velocity in the transverse plane was also calculated as representation of the dynamic movement of the trunk with respect to the pelvis. At the shoulder, the peak external rotation angle, peak internal rotation velocity, and abduction angle at the impact position were also considered. During the forward swing, the peak elbow extension velocity and the peak wrist flexion velocity were determined.

Resultant racket velocity (norm of the velocity) and racket height (barycenter of the three racket markers) were determined at the impact position. Orientation of the racket at impact is expressed with respect to the rotation axis: backward tilt (y-axis), lateral tilt (x-axis) and polar rotation (z-axis).

Only racket and center of motion variables were kept and used for the comparison between the laboratory and tennis court conditions.

\section{Statistical analysis}

Descriptive statistics (mean and standard deviation) were calculated for both groups.

For the inter-session comparison, the relative and absolute reliability of the variables were examined using an Intraclass Correlation Coefficient (ICC 2,1) and a paired Student's test, respectively. The ICC "represents the proportion of variance in a set of scores that is attributable to the true score variance" (Weir, 2005). The ICC is presented as value $[95 \% \mathrm{CI}$ low value/ $95 \% \mathrm{CI}$ high value] (Bosquet et al., 2010). The ICC 'cut-off' ranking was interpreted as poor (ICC $<0.40$ ), fair $(0.40 \leq$ ICC $<0.70)$, good $(0.70 \leq$ ICC $<0.90)$ or excellent (ICC $\geq 0.90$ ) (Coppieters et al., 2002).

For the comparisons between the laboratory and field evaluations, absolute reliability was determined with the Student's ttest for a parametric distribution and Wilcoxon tests for non-parametric distribution.

All calculations were made using Statistica 10 (Statsofts, Tulsa, OK), MedCalc 13.3.3 (MedCalc Software, Ostend, Belgium) and Excel
2013 (Microsoft, Washington, United States of America).

\section{Results}

Table 1 shows repeatability in laboratory conditions, while Table 2 shows the influence of the laboratory versus field context.

With regard to the inter-session reproducibility (Table 1), the majority of the kinetic and kinematic variables of the lower limbs presented good relative reliability (ICC $>0.7$ ). The Student's t-tests did not show a significant difference between the tests, except for "Height barycenter racket position at impact", "Racket lateral tilt at impact" and "Racket propulsion duration".

Though lower relative reliability results were observed for upper limb variables compared to lower limb variables, the dominant arm (which holds the racket) presented good ICC values $(0.70$ $\leq$ ICC < 0.90). Temporal variables expressed in seconds before impact were repeatable in the inter-session test except for the maximal external rotation, which presented a poor ICC $(<0.4)$. With a poor ICC value, accuracy of the ball seemed to be not reliable for the inter-session test. Variables of the racket were reliable except for the backward tilt and polar rotation, which presented poor results of inter-session repeatability.

Only "Height barycenter racket position at impact" highlighted a significant difference in the comparison between the laboratory and field testing (Table 2).

\section{Discussion}

Certain biomechanical variables are frequently described in the literature as performance variables for the tennis serve (Whiteside et al., 2013b) and/or to prevent injury of the tennis player (Martin et al., 2013). The goals of this study were (1) to verify the inter-session repeatability of these biomechanical variables during a tennis serve and (2) to measure the concordance of the evaluations made in a laboratory and on a tennis court. The choice of a protocol to perform a kinematic evaluation of the tennis serve depends on multiple factors, such as choice of the population, warm-up, definition of the gesture, racket type, number of attempts, localization of the test, as well as camera and marker placements (Tubez et al., 2017). 


\section{Table 1}

Inter-session reliability. ICC = Intraclass Correlation Coefficient .

The protocol was performed with 28 markers set. BW = Body Mass

\begin{tabular}{|c|c|c|c|c|c|}
\hline Variables & Unit & $\begin{array}{c}\text { Lab - test } 1 \\
(\text { Mean } \pm \text { SD })\end{array}$ & $\begin{array}{l}\text { Lab - test } 2 \\
(\text { Mean } \pm S D)\end{array}$ & t-test & ICC $[95 \% \mathrm{Cl}]$ \\
\hline \multicolumn{6}{|l|}{ Kinetic - Lower limbs } \\
\hline $\begin{array}{r}\text { Peak norm ground reaction force relative } \\
\text { to the body mass }\end{array}$ & $\begin{array}{c}\text { newto } \\
\text { n/BM }\end{array}$ & $2.07 \pm 0.38$ & $2.07 \pm 0.37$ & 0.96 & $0.98[0.93 / 0.99]$ \\
\hline \multicolumn{6}{|l|}{ Kinematic - Lower-Limbs } \\
\hline $\begin{array}{r}\text { Feet's X distance at maximal knees } \\
\text { flexion }\end{array}$ & $\mathrm{mm}$ & $263 \pm 138$ & $266 \pm 128$ & 0.77 & $0.98[0.94 / 0.99]$ \\
\hline $\begin{array}{r}\text { Feet's Y distance at maximal knees } \\
\text { flexion }\end{array}$ & $\mathrm{mm}$ & $180 \pm 82$ & $183 \pm 86$ & 0.69 & $0.96[0.87 / 0.98]$ \\
\hline Peak front knee flexion angle & deg & $65 \pm 14$ & $64 \pm 14$ & 0.51 & $0.96[0.87 / 0.99]$ \\
\hline Peak back knee flexion angle & deg & $79 \pm 11$ & $76 \pm 9$ & 0.15 & $0.78[0.40 / 0.93]$ \\
\hline $\begin{array}{r}\text { Triple extension velocity in front lower } \\
\text { limb }\end{array}$ & $\mathrm{deg} / \mathrm{s}$ & $985 \pm 202$ & $999 \pm 195$ & 0.66 & $0.88[0.63 / 0.96]$ \\
\hline $\begin{array}{r}\text { Triple extension velocity in back lower } \\
\text { limb }\end{array}$ & $\mathrm{deg} / \mathrm{s}$ & $1330 \pm 380$ & $1349 \pm 314$ & 0.52 & $0.96[0.89 / 0.99]$ \\
\hline Peak front hip vertical velocity & $\mathrm{m} / \mathrm{s}$ & $1.57 \pm 0.33$ & $1.56 \pm 0.36$ & 0.89 & $0.95[0.83 / 0.98]$ \\
\hline Peak back hip vertical velocity & $\mathrm{m} / \mathrm{s}$ & $2.22 \pm 0.27$ & $2.19 \pm 0.25$ & 0.09 & $0.99[0.95 / 0.99]$ \\
\hline \multicolumn{6}{|l|}{ Kinematic - Trunk \& Pelvis } \\
\hline $\begin{array}{r}\text { Peak trunk-pelvis girdles separation } \\
\text { angle (transversal) }\end{array}$ & deg & $20.6 \pm 11.3$ & $19.0 \pm 9.1$ & 0.52 & $0.72[0.25 / 0.92]$ \\
\hline $\begin{array}{r}\text { Peak trunk-pelvis girdles tilt angle } \\
\text { (frontal) }\end{array}$ & deg & $14.5 \pm 9.8$ & $17.6 \pm 9.6$ & 0.12 & $0.91[0.73 / 0.97]$ \\
\hline $\begin{array}{r}\text { Peak trunk-pelvis girdles twist velocity } \\
\text { (transversal) }\end{array}$ & $\operatorname{deg} / \mathrm{s}$ & $350.6 \pm 163.3$ & $358.2 \pm 161.6$ & 0.84 & $0.70[0.27 / 0.90]$ \\
\hline \multicolumn{6}{|l|}{ Kinematic - Serving arm } \\
\hline Peak shoulder external rotation angle & deg & $117.5 \pm 19.4$ & $120.3 \pm 19.3$ & 0.48 & $0.83[0.48 / 0.95]$ \\
\hline Peak shoulder internal rotation velocity & $\mathrm{deg} / \mathrm{s}$ & $1667 \pm 381$ & $1641 \pm 576$ & 0.82 & $0.72[0.28 / 0.91]$ \\
\hline Peak elbow extension velocity & $\mathrm{deg} / \mathrm{s}$ & $1320 \pm 208$ & $1367 \pm 242$ & 0.28 & $0.85[0.53 / 0.96]$ \\
\hline Peak wrist flexion velocity & $\mathrm{deg} / \mathrm{s}$ & $921 \pm 145$ & $967 \pm 208$ & 0.37 & $0.89[0.67 / 0.96]$ \\
\hline Shoulder abduction angle at impact & deg & $105 \pm 13$ & $106 \pm 8$ & 0.39 & $0.71[0.22 / 0.91]$ \\
\hline \multicolumn{6}{|l|}{ Kinematic - Racket } \\
\hline Resultant racket velocity at impact & $\mathrm{m} / \mathrm{s}$ & $38.9 \pm 2.5$ & $38.6 \pm 3.5$ & 0.74 & $0.90[0.69 / 0.97]$ \\
\hline $\begin{array}{r}\text { Height barycenter racket position at } \\
\text { impact }\end{array}$ & $\mathrm{m}$ & $2.643 \pm 0.113$ & $2.664 \pm 0.118$ & 0.04 & $0.97[0.89 / 0.99]$ \\
\hline Racket backward tilt at impact & deg & $86.0 \pm 4.5$ & $87.3 \pm 3.2$ & 0.48 & $-0.38[-0.77 / 0.22]$ \\
\hline Racket lateral tilt at impact & deg & $75.3 \pm 4.7$ & $72.7 \pm 3.4$ & 0.01 & $0.74[0.31 / 0.91]$ \\
\hline Racket polar rotation at impact & deg & $7.8 \pm 5.1$ & $6.4 \pm 2.7$ & 0.48 & $0.01[-0.57 / 0.58]$ \\
\hline \multicolumn{6}{|l|}{ Temporal } \\
\hline $\begin{array}{r}\text { Center of Motion minimal vertical } \\
\text { position }\end{array}$ & $\mathrm{s}$ & $-0.38 \pm 0.08$ & $-0.38 \pm 0.07$ & 0.89 & $0.95[0.85 / 0.99]$ \\
\hline $\begin{array}{r}\text { High racket position point (trophy } \\
\text { position or HRP) }\end{array}$ & $\mathrm{s}$ & $-0.34 \pm 0.08$ & $-0.35 \pm 0.09$ & 0.20 & $0.98[0.95 / 0.99]$ \\
\hline Low racket position point (LRP) & $\mathrm{s}$ & $-0.12 \pm 0.01$ & $-0.12 \pm 0.01$ & 0.69 & $0.77[0.39 / 0.93]$ \\
\hline Maximal shoulder external rotation & $\mathrm{s}$ & $-0.10 \pm 0.02$ & $-0.12 \pm 0.02$ & 0.13 & $0.21[-0.45 / 0.72]$ \\
\hline Racket propulsion duration & $\mathrm{s}$ & $0.19 \pm 0.04$ & $0.19 \pm 0.04$ & 0.04 & $0.99[0.94 / 0.99]$ \\
\hline Leg drive duration & $\mathrm{s}$ & $0.26 \pm 0.07$ & $0.26 \pm 0.06$ & 0.92 & $0.94[0.82 / 0.98]$ \\
\hline \multicolumn{6}{|l|}{ Accuracy } \\
\hline $\begin{array}{r}\text { Number of points reached per serve } \\
\text { (maximum }=6 \text { points) }\end{array}$ & point & $1.8 \pm 0.5$ & $2.1 \pm 0.7$ & 0.27 & $0.26[-0.32 / 0.70]$ \\
\hline
\end{tabular}


Table 2

Field - laboratory comparison. The protocol was performed with 4 markers set.

\begin{tabular}{|c|c|c|c|c|}
\hline Variables & Unit & $\begin{array}{l}\text { Field test } \\
(\text { Mean } \pm \text { SD) }\end{array}$ & $\begin{array}{c}\text { Lab test } \\
(\text { Mean } \pm \mathrm{SD})\end{array}$ & t-test \\
\hline \multicolumn{5}{|l|}{ Kinematic - Racket } \\
\hline Resultant racket maximal velocity at impact & $\mathrm{m} / \mathrm{s}$ & $38.5 \pm 3.4$ & $38.7 \pm 3.3$ & 0.67 \\
\hline Height barycenter racket position at impact & $\mathrm{m}$ & $\begin{array}{c}2.717 \pm \\
0.108\end{array}$ & $\begin{array}{c}2.692 \pm \\
0.102\end{array}$ & 0.03 \\
\hline Racket backward tilt at impact & deg & $87.2 \pm 3.9$ & $87.9 \pm 4.0$ & 0.61 \\
\hline Racket lateral tilt at impact & $\operatorname{deg}$ & $75.3 \pm 5.6$ & $75.1 \pm 5.6$ & 0.88 \\
\hline Racket polar rotation at impact & $\operatorname{deg}$ & $3.5 \pm 6.1$ & $6.7 \pm 6.1$ & 0.08 \\
\hline \multicolumn{5}{|l|}{ Temporal } \\
\hline Center of Motion minimal vertical position & $\mathrm{s}$ & $-0.39 \pm 0.07$ & $-0.38 \pm 0.06$ & 0.41 \\
\hline High racket position point (trophy position or HRP) & $\mathrm{s}$ & $-0.32 \pm 0.14$ & $-0.31 \pm 0.12$ & 0.68 \\
\hline Low racket position point (LRP) & $\mathrm{s}$ & $-0.13 \pm 0.03$ & $-0.12 \pm 0.02$ & 0.43 \\
\hline Racket propulsion duration & $\mathrm{s}$ & $0.20 \pm 0.09$ & $0.20 \pm 0.08$ & 0.99 \\
\hline \multicolumn{5}{|l|}{ Accuracy } \\
\hline $\begin{array}{r}\text { Number of points reached per serve (maximum }=6 \\
\text { points) }\end{array}$ & point & $1.9 \pm 0.7$ & $2.1 \pm 0.6$ & 0.533 \\
\hline
\end{tabular}
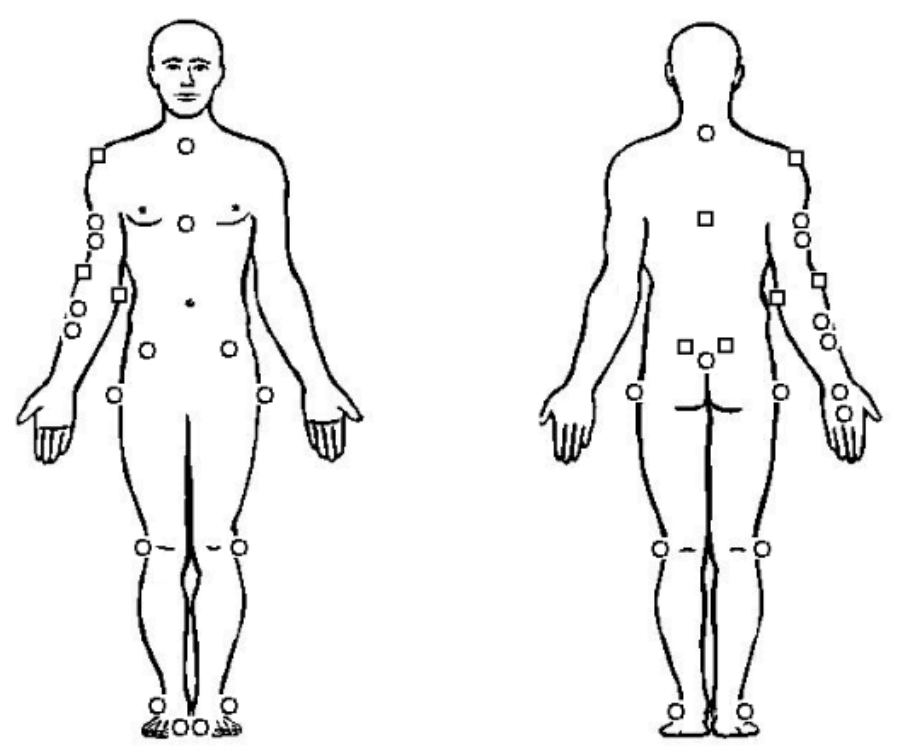

Figure 1

Representation of the marker placement. Circles represent markers and squares represent supplement anatomical points. 

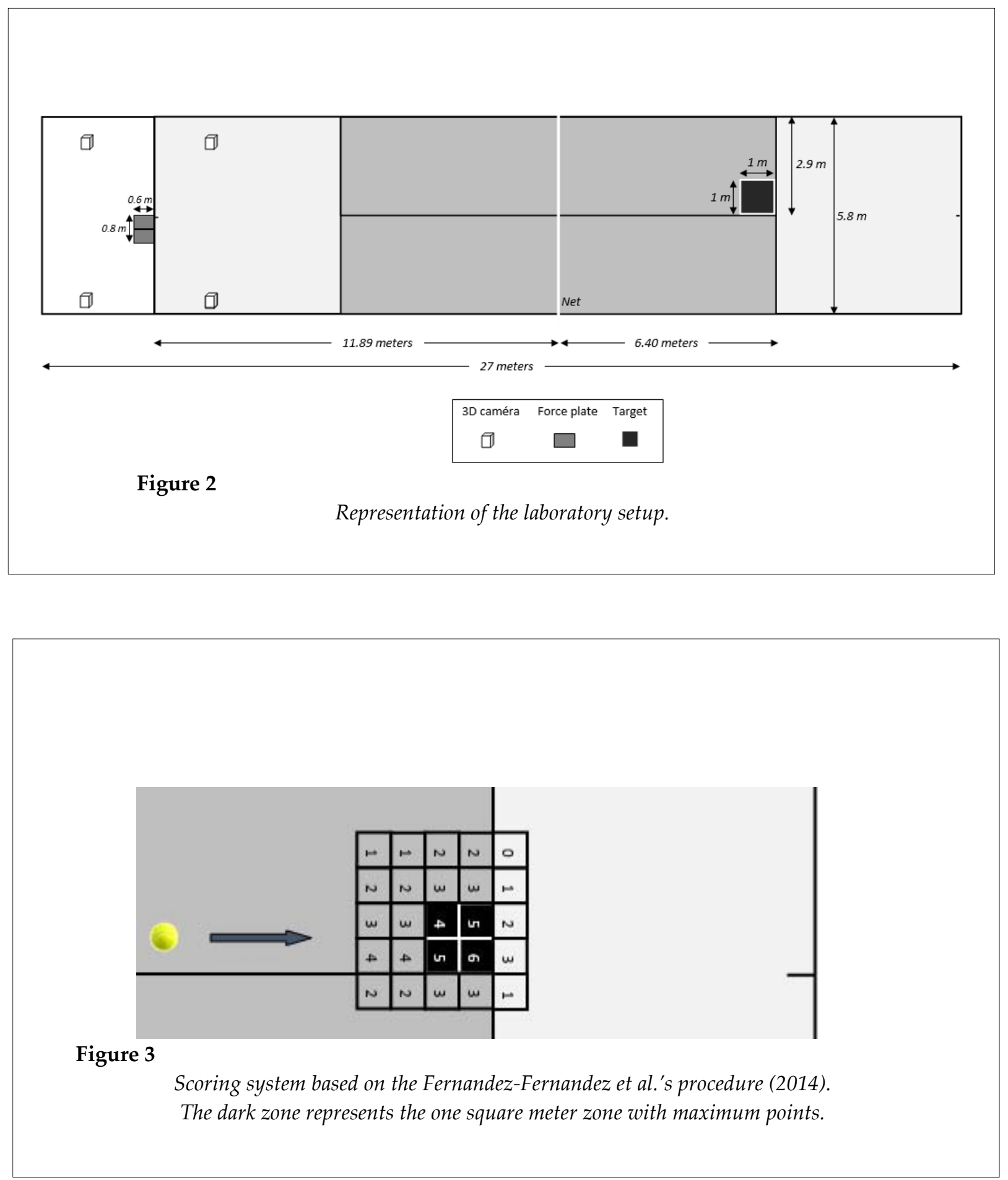

This study used a protocol in accordance with the current literature on tennis serve evaluation (Martin et al., 2014; Tubez et al., 2017; Whiteside et al., 2013a). High-level players in their age categories were proposed to perform 15 first serves in the "T area" of the deuce diagonal in a laboratory tennis court.

The tennis serve is a succession of phases determined by various positions. Giblin and colleagues (2017) proposed a model with three phases (preparation, propulsion and forward swing) and four key events (ball release, trophy position, racket low point and impact). Occurrence of these instants and phases seems to be reliable for inter-session tests and the laboratory versus field comparison (trophy position or HRP, racket low point, propulsion and forward swing). 
The lower limbs play an essential role in the biomechanics of the serve (Bonnefoy et al., 2009). Foot technique (foot-up versus foot-back) and kinetic peak ground reaction forces are described as performance variables in the literature (Girard et al., 2005). An excellent relative inter-session reliability is observed for this kinetic variable. Although this step has already been taken in a tennis court laboratory (Martin et al., 2014), a force plate integrated into an official tennis court is hardly imaginable, and even less so in a context of competition. The laboratory context with a platform integrated in the ground shows a real interest for the tennis serve kinetic evaluation. Leg drive causes lower limb triple extension and an increase in the ground reaction forces (Girard et al., 2007; Whiteside et al., 2013b). The triple extension of the legs creates a back hip upward velocity, which is described as an essential factor in the transmission of energy from legs to upper body segments (Sweeney et al., 2012; Whiteside et al., 2013b). These two variables present high inter-session reliability.

The energy flow is transmitted along the kinetic chain from the lower limbs to the upper limbs through the trunk (Kibler, 2014; Martin et al., 2014). Lower-limb kinematics measurements seem less prone to variations than trunk and dominant arm variables. It is likely that adaptations and compensations are greater at the end of the kinetic chain (upper limbs) than at the start (lower limbs). The results show gestural and temporal modifications of the kinematics. According to Giblin and colleagues (2017), the player adapts the serve kinematics to the ball trajectory during the toss in order to coincide with the final ball impact location and timing.

Trunk rotations are paramount to the development of high hitting speeds (Elliott, 2006). Separation girdles amplitude and velocities are factors to be considered in the transmission and generation of the energy flow (Whiteside et al., 2013a). The trunk, the link between the lower and the upper limb, is also a source of injuries that is important to prevent (Martin et al., 2013; Tubez et al., 2015). The trunk variables observed in this study present good inter-session reliability.

The dominant upper limb is the last part in the transmission of the energy flow from the legs to the racket during the tennis serve. Similar to knee flexion and trunk rotation, shoulder external rotation prepares the transmission and the generation of energy in the kinetic chain. Maximal shoulder external rotation is described for performance and injury prevention (Elliott et al., 2003). It has been also shown that internal shoulder rotation velocity is a very important variable in the production of ball speed (contribution greater than $50 \%$ of the racket velocity at impact) (Marshall and Elliott, 2000). Elbow extension and wrist flexion are the last motions that generate and transmit energy to the racket. All of these performance variables present good inter-session reliability. Impact height is also crucial to perform an efficient tennis serve or an ace (Whiteside and Reid, 2016). In this protocol, the barycenter (center of the three racket markers) height is used as an approximation of the ball impact height. This approach is also used in the literature (Bonnefoy et al., 2009). Similar results are observed in comparison with the literature (Chow et al., 2003) and excellent reliability is observed in the inter-session procedure. We observed higher impact height on the tennis court than in the laboratory. The tests were performed in a laboratory with a lower ceiling $(4.00 \mathrm{~m})$ than on the tennis court $(<12.00 \mathrm{~m})$. The players might have been influenced by this context. However, limited influence on the kinematics of gestures and the serve performance was observed.

Ball velocity is also an essential variable of the performance of a tennis serve (Bahamonde, 2000). In this study, the racket velocity variable was measured rather than the ball velocity, because it represents more directly the player performance excluding the influence of the racket and the string bed. The results of this study were similar to reports in the literature (Tanabe and Ito, 2007) and showed that racket velocity at impact presented reliable inter-session results. Similar racket velocity results between the laboratory and the tennis court conditions were observed, reflecting the absence of an influence of the laboratory conditions on the racket velocity at impact. Direction and ball effect are partly determined by the racket orientation at impact (Cross, 2011). Height of the toss is another determinant of the ball direction and ball rotation (effect of the ball). At impact position, only the lateral tilt seemed to be reliable for the intersession test. The serve is a closed skill and the 
player has maximum control on the task. However, it does not mean that these serves were exactly the same among multiple trials (Reid et al., 2010). The players need to adapt the racket position according to the ball toss for each serve, and the racket position at impact varies from one serve to another. It seems rational to observe variation of the racket orientation at impact. These variations should be all more important for the more distal segments of the kinetic chain. Furthermore, the racket moves fast at impact time. The acquisition frame rate was $200 \mathrm{~Hz}$ and was potentially too low to exactly determine the impact of the ball with the racket. It would be interesting to study the accuracy of the impact instant for several frequencies of acquisition.

We developed a measuring method to evaluate the accuracy of the ball's landing location. The procedure is comparable to the Fernandez-Fernandez et al.'s method (FernandezFernandez et al., 2014). With this method, although accuracy was not determined to be significantly different between tests, poor reliability was observed. Previous experience may lead the player to prioritize accuracy over velocity. This theory is supported by the speed accuracy trade-off (SAT) phenomenon encountered in tennis (Chow et al., 2003) and could partly explain the poor reliability of the inter-session test. We also noted no significant difference concerning the ball accuracy between laboratory and field conditions. Thus it seems that the unfamiliar visual environment (distances and space) encountered in the laboratory did not influence the accuracy.

Technology is increasingly available to the public and provides feedback to tennis players to help them improve their performance and reduce their risk of injury. Certain of these tools are complex and are mostly found in laboratories, while others, being simpler and cheaper, are widespread and can be used by most in field studies. These new tools are often portable (e.g., accelerometer and electromyography) and may provide an alternative to the laboratory tests in the future.

Our study has several limitations. Firstly, the laboratory ceiling may have had a limited influence on the racket impact height. Secondly, adding four markers on the player and cameras on the court still slightly differs from the real competition game. Thirdly, the Hawthorne effect has to be considered in a laboratory study. However, its influence in our approach was limited because we compared two identical laboratory tests. Moreover, for the laboratory versus the field comparison, the players are aware, in both situations, of participating in an experiment.

To conclude, the present study consists of evaluating the reproducibility and pertinence of a biomechanical evaluation of the tennis serve in a laboratory. The studied variables had been described in the literature as variables related to performance and/or injury prevention of the tennis serve. We observed that the majority of these variables had excellent inter-session relative reliability. We also showed that the impact of the laboratory versus field context on the player's serve was limited. Overall, the present study demonstrated that tests performed in a laboratory can be used to evaluate a tennis player' serve.

\section{Acknowledgements}

The authors wish to thank the Wallonia-Brussels Federation for its support.

\section{References}

Bahamonde R. Changes in angular momentum during the tennis serve. J Sports Sci, 2000; 18(8): 579-592

Benedetti MG, Marshall RN, Elliott BC. Long-axis rotation: the missing link in proximal-to-distal segmental sequencing. J Sports Sci, 2000; 18(4): 247-254

Bonnefoy A, Slawinski J, Riquet A, Leveque JM, Miller C, Cheze L. Rotation sequence is an important factor in shoulder kinematics. Application to the elite players' flat serves. J Biomech, 2010; 43(10): 2022-2025

Bonnefoy A, Slawinski J, Leveque JM, Riquet A, Miller C. Relationship between the vertical racquet head height and the lower limb motions of elite players' flat serve. Comput Methods Biomech Biomed Engin, 2009; 12(sup1): 55-57 
Bosquet L, Maquet D, Forthomme B, Nowak N, Lehance C, Croisier JL. Effect of the lengthening of the protocol on the reliability of muscle fatigue indicators. Int J Sports Med, 2010; 31(2): 82-88

Chow J, Carlton L, Lim Y, Chae WS, Shim JH, Kuenster AF, Kokubun K. Comparing the pre- and postimpact ball and racquet kinematics of elite tennis players' first and second serves: a preliminary study. J Sports Sci, 2003; 21(7): 529-537

Coppieters M, Stappaerts K, Janssens K, Jull G. Reliability of detecting "onset of pain" and "submaximal pain" during neural provocation testing of the upper quadrant. Physiother Res Int, 2002; 7(3): 146-156

Cross R. The kick serve in tennis. Sport. Technol, 2011; 4: 19-28

Elliott B. Biomechanics and tennis. Br J Sports Med, 2006; 40(5): 392-396

Elliott B, Fleisig G, Nicholls R, Escamilia R. Technique effects on upper limb loading in the tennis serve. J Sci Med Sport, 2003; 6(1): 76-87

Fernandez-Fernandez J, Ulbricht A, Ferrauti A. Fitness testing of tennis players: How valuable is it? $\mathrm{Br} J$ Sports Med, 2014; 48: 1-12

Ferrauti A, Kinner V, Fernandez-Fernandez J. The Hit \& Turn Tennis Test: an acoustically controlled endurance test for tennis players. J Sports Sci, 2011; 29(5): 485-494

Gamage S, Lasenby J. New least squares solutions for estimating the average centre of rotation and the axis of rotation. J Biomech, 2002; 35(1): 87-93

Giblin G, Whiteside D, Reid M. Now you see, now you don't ... the influence of visual occlusion on racket and ball kinematics in the tennis serve. Sport Biomech, 2017; 16(1): 23-33

Girard O, Micallef JP, Millet G. Influence of restricted knee motion during the flat first serve in tennis. J Strength Cond Res, 2007; 21(3): 950-957

Girard O, Micallef JP, Millet G. Lower-limb activity during the power serve in tennis: Effects of performance level. Med Sci Sports Exerc, 2005; 37: 1021-1029

Göktepe A, Ak E, Söğüt M, Karabörk H, Korkusuz F. Joint angles during successful and unsuccessful tennis serves kinematics of tennis serve. Eklem Hastalik Cerrahisi, 2009; 20(3): 156-160

Ida $\mathrm{H}$, Kusubori S, Ishii M. Kinematics and kinetics of the racket-arm during the soft-tennis smash under match conditions. J Appl Biomech, 2005; 21(4): 334-347

Karsten B, Jobson S, Hopker J, Jimenez A, Beedie C. High agreement between laboratory and field estimates of critical power in cycling. Int J Sports Med, 2014; 35(4): 298-303

Kibler W. Understanding the kinetic chain. Aspetar Sport Med J, 2014; 3(Targered Topic): 492-497

Kovacs M, Ellenbecker T. An 8-Stage Model for Evaluating the Tennis Serve: Implications for Performance Enhancement and Injury Prevention. Sport Heal A Multidiscip Approach, 2011; 3: 504-513

Manca M, Leardini A, Cavazza S, Ferraresi G, Marchi P, Zanaga E. Repeatability of a new protocol for gait analysis in adult subjects. Gait and Posture, 2010; 32(2): 282-284

Martin C, Bideau B, Bideau N, Nicolas G, Delamarche P, Kulpa R. Energy Flow Analysis During the Tennis Serve: Comparison Between Injured and Noninjured Tennis Players. Am J Sports Med, 2014; 42(11): 2751-2760

Martin C, Kulpa R, Ropars M, Delamarche P, Bideau B. Identification of temporal pathomechanical factors during the tennis serve. Med Sci Sports Exerc, 2013; 45(11): 2113-2119

Mullineaux DR, Bartlett RM, Bennett S. Research design and statistics in biomechanics and motor control. J Sports Sci, 2001; 19(10): 739-760

Reid M, Whiteside D, Elliott B. Effect of skill decomposition on racket and ball kinematics of the elite junior tennis serve. Sports Biomech, 2010; 9(4): 296-303

Schwartz C, Croisier JL, Rigaux E, Brüls O, Denoël V, Forthomme B. Gender effect on the scapular 3D posture and kinematic in healthy subjects. Clin Physiol Funct Imaging, 2016; 36(3): 188-196

Smith L, Kensrud J. Field and laboratory measurements of softball player swing speed and bat performance. 
Sport. Eng, 2014; 17: 75-82

Sweeney M, Reid M, Elliott B. Lower Limb and Trunk Function in the High Performance Tennis Serve. Asian J Exerc, 2012; 9(1): 13-21

Tanabe S, Ito A. A three-dimensional analysis of the contributions of upper limb joint movements to horizontal racket head velocity at ball impact during tennis serving. Sport Biomech, 2007; 6(3): 418-433

Tubez F, Forthomme B, Croisier J-L, Brüls O, Denoël V, Schwartz C. Factors to be considered to perform a kinematic evaluation of the tennis serve. J Med Sci Tennis, 2017; 22: 12-16

Tubez F, Forthomme B, Croisier J, Cordonnier C, Brüls O, Denoël V, Berwart G, Joris M, Grosdent S, Schwartz C. Biomechanical Analysis of Abdominal Injury in Tennis Serves. A Case Report. J Sport Sci Med, 2015; 14: 402-412

Weir JP. Quantifying Test-Retest Reliability Using the Intraclass Correlation Coefficient and the SEM. $J$ Strength Cond Res, 2005; 19(1): 231

Whiteside D, Elliott B, Lay B, Reid M. A kinematic comparison of successful and unsuccessful tennis serves across the elite development pathway. Hum Mov Sci, 2013a; 32(4): 822-835

Whiteside D, Elliott B, Lay B, Reid M. The effect of age on discrete kinematics of the elite female tennis serve. J Appl Biomech, 2013b; 29(5): 573-582

Whiteside D, Reid M. Spatial characteristics of professional tennis serves with implications for serving aces: A machine learning approach. J Sports Sci, 2016; 35(7): 648-654

Wu G, van der Helm F, Veeger H, Makhsous M, Van Roy P, Anglin C, Nagels J, Karduna A, McQuade K, Wang X, Werner F, Buchholz B. ISB recommendation on definitions of joint coordinate systems of various joints for the reporting of human joint motion - Part II: shoulder, elbow, wrist and hand. J Biomech, 2005; 38(5): 981-992

Wu G, Siegler S, Allard P, Kirtley C, Leardini A, Rosenbaum D, Whittle M, D'Lima D, Cristofolini L, Witte $\mathrm{H}$, Schmid O, Stokes I. ISB recommendation on definitions of joint coordinate system of various joints for the reporting of human joint motion - Part I: ankle, hip, and spine. J Biomech, 2002; 35(4): 543-548

\section{Corresponding author:}

\section{François Tubez}

Laboratory of Human Motion Analysis - University of Liège (Belgium)

Quartier Polytech 1, Allée de la Découverte 13C 4000 Liège (Belgium)

Phone: +32 (0) 497/20.69.55

E-mail: Francois.Tubez@ulg.ac.be 